\title{
Replace the Content in e-Abacus Diagram
}

\author{
Ammar Seddiq Mahmood \\ Department of Mathematics, College of Education for Pure Science, University of Mosul, Mosul, Iraq \\ Email: asmahmood65@yahoo.fr, asmahmood65@uomosul.edu.iq
}

How to cite this paper: Mahmood, A.S. (2020) Replace the Content in e-Abacus Diagram. Open Access Library Journal, 7: e6211.

https://doi.org/10.4236/oalib.1106211

Received: March 5, 2020

Accepted: April 11, 2020

Published: April 14, 2020

Copyright $\odot 2020$ by author(s) and Open Access Library Inc.

This work is licensed under the Creative Commons Attribution International License (CC BY 4.0).

http://creativecommons.org/licenses/by/4.0/

\begin{abstract}
This research is based on the idea of replacing what the sites contain in the e-abacus diagram with each other and then representing the new diagram (derived from the original diagram) and knowing the value of its partition (as a type of hidden coding of the origin of the diagram or in the sense of the opposite diagram).
\end{abstract}

\section{Subject Areas}

Mathematics, Partition Theory

\section{Keywords}

Partition Theory, Encoding, e-Abacus Diagram

\section{Introduction}

Let $r$ be a non-negative integer. A partition $\delta=\left(\delta_{1}, \delta_{2}, \cdots, \delta_{n}\right)$ of $r$ is a sequence of non-negative integers such that $|\delta|=\sum_{j=1}^{n} \delta_{j}=r, \forall j \geq 1$ and $\delta_{j} \geq \delta_{j+1}$. Fix $\delta$ is a partition of $r$, choosing an integer $b$ greater than or equal to the number of parts of $\delta$ and defining $\beta_{i}=\delta_{i}+b-i, 1 \leq i \leq b$. The set $\left\{\beta_{1}, \beta_{2}, \cdots, \beta_{b}\right\}$ is said to be the set of $\beta$-numbers for $\delta$, see [1]. Let $e$ be a positive integer number greater than or equal to 2 , we can represent $\beta$-numbers by a diagram called e-abacus diagram (Table 1).

Table 1. e-Abacus diagram.

\begin{tabular}{cccc}
\hline Run.1 & Run.2 & $\ldots$ & Run.e \\
\hline 0 & 1 & $\ldots$ & $e-1$ \\
$2 e$ & $e+1$ & $\ldots$ & $2 e-1$ \\
$:$ & $2 e+1$ & $\ldots$ & $3 e-1$ \\
\hline
\end{tabular}


Where every $\beta$ will be represented by a bead ( $\boldsymbol{\square})$ and the rest of the sites by $(-)$, which takes its location e-abacus diagram [2]. In this work, we will try to present the opposite diagram to the main diagram and note all the changes that occurred from that change. This issue is being discussed for the first time.

\section{Replace the Content}

In this section, we will present the idea of replacing what the sites contain in the first main $e$-abacus diagram [3], with the opposite of what it contains of a ( $\mathbf{\square})$ or $(-)$ and then represent the new diagram to know the value of partition after the exchange and denoted by $e^{r}$-abacus diagram (Figures 1-3). For example, if $\delta=(6,6,5,5,5,3,1,1)$, then

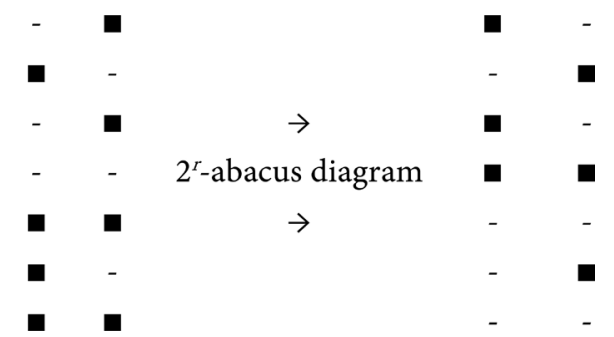

Figure 1. $2^{r}$-abacus diagram $=(6,3,3,2,2)$.

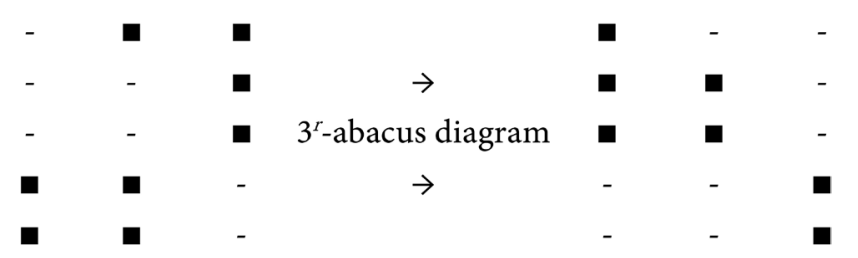

Figure 2. $3^{r}$-abacus diagram $=(8,6,3,3,2,2)$.

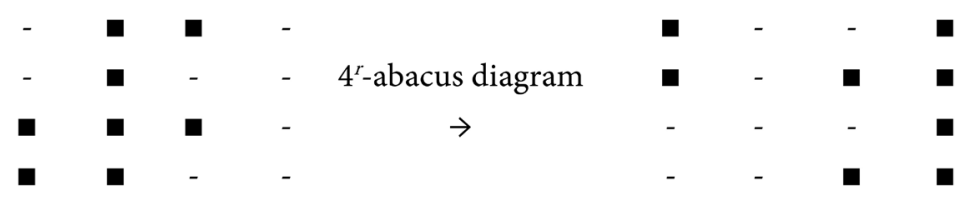

Figure 3. $4^{r}$-abacus diagram $=(8,8,6,3,3,2,2)$.

Here we note that for one case of any partition it turned into several formats of the partitions in the case of $e^{r}$-abacus diagram and perhaps to other formats in the case of different $e$, as if it becomes a new coding completely difficult (detecting) easily. We will try hard to explain this phenomenon and clarify its implications.

\section{Explanation of This Phenomenon}

In fact, the important thing here is the location of $\left(\delta_{1}\right)$ according to its presence in any row $k$ and any column $l$ depending on the value of $e$, by the following equations:

$$
\text { Location of } \delta_{1}=\delta_{1}+((\text { no. of parts of } \delta)-1)
$$

which is exactly equal to 


$$
\text { Location of } \delta_{1}=(k-1) e+(l-1)
$$

where $k$ is a positive integer and $1 \leq l \leq e$.

From the above example,

The reason for the importance (Location of $\delta_{1}$ ) is to know when we stand in the representation of the original diagram as well as in the new diagram after the replacement.

This is on the one hand, on the other hand if any ( - ) site is in the last place at l, specifically in the last row $k$, then this means that this will turn into a (-) after the replacement. But if you are not in the last column $l$, then the sites that follow that place will turn into beads after the replacement, then the number of sites available to us within the last row.

Returning to Table 2, in the case $e=2$ there is no addition any ( $\square$ ), while we will add (one ( $\square$ ), two ( $\square$ ) and one ( $\square)$ ) when ( $e=3, e=4$ and $e=5$ ) respectively after the replacement. We have to fully understand the behavior of this type, depending on the value of $e$, the representation mechanism after the exchange and for this we will take the following example:

The question now is, can we know the value of partition immediately after the replacement of the main partition? The answer is YES, but we have to use $e$ and $I$ as follows:

In order to facilitate the solution, we will take the following steps:

Table 2. The location of $\delta_{1}$.

\begin{tabular}{ccccccccccccc}
\hline$\delta_{1}$ & Location of $\delta_{1}$ & & \multicolumn{2}{c}{$e=2$} & & $e=3$ & & \multirow{2}{*}{$e=4$} & \multicolumn{2}{c}{$e=5$} \\
\hline & & $k$ & $l$ & $k$ & $l$ & $k$ & $l$ & $k$ & $l$ \\
6 & 13 & 7 & 2 & 5 & 2 & 4 & 2 & 3 & 4 \\
\hline
\end{tabular}

Table 3. The mechanism.

\begin{tabular}{|c|c|c|c|c|c|}
\hline$\delta$ & $\begin{array}{c}\delta_{1} \text { by } \\
\text { Equation (1) }\end{array}$ & $\begin{array}{c}\delta_{1} \text { by } \\
\text { Equation (2) }\end{array}$ & $k$ & 1 & $e^{r} \quad$-partition \\
\hline \multirow{10}{*}{$(7,5,5,1,1,1)$} & \multirow{10}{*}{12} & $e=2,6(2)+0$ & 7 & 1 & $(6,5,5,3,3,3,3)$ \\
\hline & & $e=3,4(3)+0$ & 5 & 1 & $(6,6,5,5,3,3,3,3)$ \\
\hline & & $e=4,3(4)+0$ & 4 & 1 & $(6,6,6,5,5,3,3,3,3)$ \\
\hline & & $e=5,2(5)+2$ & 3 & 3 & $(6,6,5,5,3,3,3,3)$ \\
\hline & & $e=6,2(6)+0$ & 3 & 1 & $(6,6,6,6,6,5,5,3,3,3,3)$ \\
\hline & & $e=7,1(7)+5$ & 2 & 6 & $(6,5,5,3,3,3,3)$ \\
\hline & & $e=8,1(8)+4$ & 2 & 5 & $\left(6^{3}, 5,5,3,3,3,3\right)$ \\
\hline & & $e=9,1(9)+3$ & 2 & 4 & $\left(6^{5}, 5,5,3,3,3,3\right)$ \\
\hline & & $:$ & $:$ & $:$ & $:$ \\
\hline & & $e>13,0(e)+13$ & 1 & 13 & $\left(6^{e-13}, 5,5,3,3,3,3\right)$ \\
\hline
\end{tabular}


1) We write the main partition from small to large.

2) We take the first value from first step and note how many times it will give us the origin of the swapped charge raised to the exponent, which is the difference between it and the value that follows and which is not equal at all.

3) We repeat the second step on the new different value and know its number with the addition of it (to the substitution value) and always up to the exponent (which is the difference between this value and the one that follows it and is not equal to the amount) up to the last value where in this case we have to go to step 4.

4) When $l=e$, we stand at the third step, and if $l<e$ then we repeat the third step and raise it to exponent, which is how much is the difference between $e$ and l.

To illustrate these four steps, we will refer to the example in Table 3:

Step 1: $(1,1,1,5,5,7)$

Step 2: The number of $(1,1,1)$ equal to 3 , uploaded to $4 ;(5-1=4)$, then $3^{4}$ or $(3,3,3,3)$

Step 3: The number of $(5,5)$ equal to 2 (plus to 3 of step 2, then 5), uploaded to $2 ;(7-5=2)$, then $(5,5)$ or $5^{2}$

\section{Step 4:}

\begin{tabular}{ccc}
\hline$e$ & 1 & Value of partition \\
\hline 2 & 1 & $6^{2-1}=6$ \\
3 & 1 & $6^{3-1}=6^{2}$ \\
4 & 1 & $6^{4-1}=6^{3}$ \\
5 & 3 & $6^{5-3}=6^{2}$ \\
6 & 1 & $6^{6-1}=6^{5}$ \\
7 & 6 & $6^{7-6}=6$ \\
8 & 5 & $6^{8-5}=6^{3}$ \\
9 & 4 & $6^{9-4}=6^{5}$ \\
10 & 3 & $6^{10-3}=6^{7}$ \\
11 & 2 & $6^{11-2}=6^{9}$ \\
12 & 1 & $6^{12-1}=6^{11}$ \\
13 & 13 & $6^{13-13}$ is no appear \\
\hline
\end{tabular}

Now, if we take another example, $=(8,8,8,7,4,4,2,2,2,2,1)$ :

Location of $\omega_{1}=18$, and if we take $e=5,7$ and 12 respectively, then we have Table 4.

Then the value of partitions after the replacement (Figure 4 and Figure 5) are:

Step 1: $(1,2,2,2,2,4,4,7,8,8,8)=\left(1,2^{4}, 4^{2}, 7,8^{3}\right)$

Step 2: $1^{1}$ 
Table 4. The mechanism of $\omega$.

\begin{tabular}{ccccccccccc}
\hline \multirow{2}{*}{$\omega_{1}$} & Location of $\omega_{1}$ & & \multicolumn{2}{c}{$e=5$} & & \multicolumn{2}{c}{$e=7$} & \multicolumn{2}{c}{$e=12$} \\
\cline { 3 - 10 } & & $k$ & & $l$ & $k$ & $l$ & $k$ & 1 \\
\hline 8 & 18 & & 4 & 4 & 3 & 5 & 2 & 7 \\
\hline
\end{tabular}

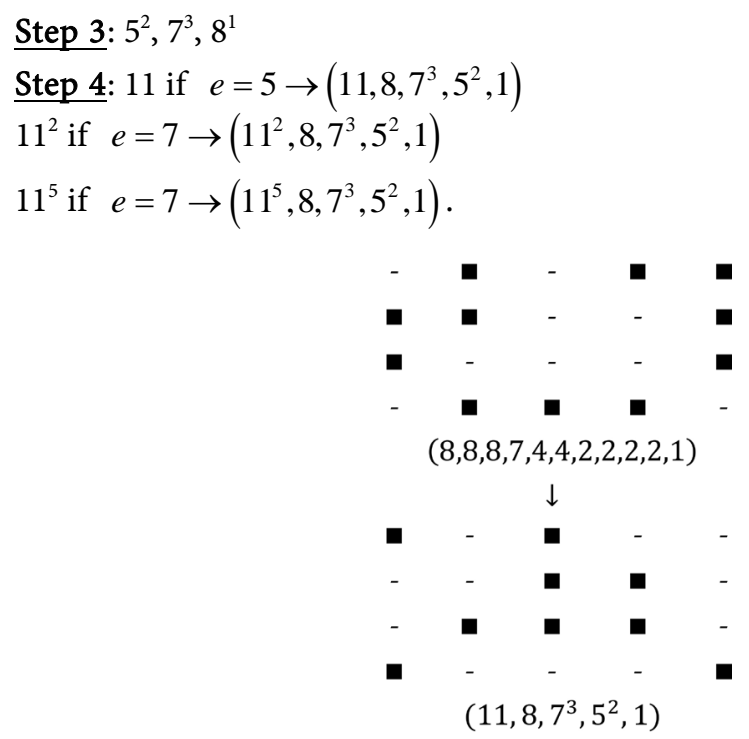

Figure 4. The replacement of $(8,8,8,7,4,4,2,2,2,2,1)$ if $e=5$.

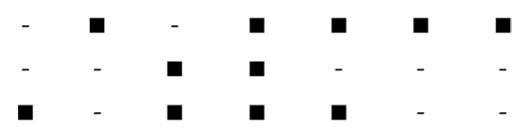

$(8,8,8,7,4,4,2,2,2,2,1)$

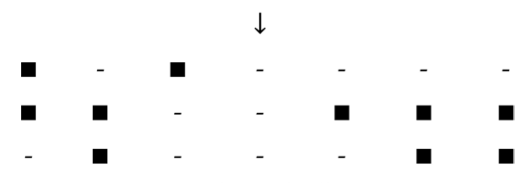

$\left(11^{2}, 8,7^{3}, 5^{2}, 1\right)$

Figure 5. The replacement of $(8,8,8,7,4,4,2,2,2,2,1)$ if $e=7$.

\section{Conclusions}

1) Through one partition, we will have a completely unfinished number of replaced partition, which will make it difficult to fully reveal the origin of the partition.

2) A very new style that was not previously discussed in coding the partition.

3) No matter how small the code is, the new encoding will make it difficult to find the original.

4) It is possible to apply this method to the types that have already been talked about, see [4] [5] [6] [7].

\section{Conflicts of Interest}

The author declares no conflicts of interest regarding the publication of this paper. 


\section{References}

[1] Mathas, A. (1999) Iwahori-Hecke Algebras and Schur Algebras of the Symmetric Group. University Lecture Series, 15, 188 p. http://doi.org/10.1090/ulect/015/02

[2] James, G. (1978) Some Combinatorial Results Involving Young Diagrams. Mathematical Proceedings of the Cambridge Philosophical Society, 83, 1-10. http://doi.org/10.1017/S0305004100054220

[3] Mahmood, A.S. (2011) On the Intersection of Young's Diagrams Core. Journal of Education and Science, 24, 149-157. http://doi.org/10.33899/edusj.1999.58795

[4] Mahmood, A.B. and Mahmood, A.S. (2019) Secret-Word by E-Abacus Diagram I. Iraqi Journal of Science, 60, 638-646.

http://www.researchgate.net/publication/332058738

[5] Mahmood, A.B. and Mahmood, A.S. (2019) Secret-Text by E-Abacus Diagram II. Iraqi Journal of Science, 60, 840-846. http://www.researchgate.net/publication/332786557

[6] Shareef, R.J. and Mahmood, A.S. (2019) The Movement of Orbits and Their Effect on the Encoding of Letters in Partition Theory. Open Access Library Journal, 6, 1-7. http://doi.org/10.4236/oalib.1105834

[7] Sami, H.H. and Mahmood, A.S. (2020) Encoding Syriac Letters in Partition Theory Using Extended Vigenere Cipher. Eastern-European Journal of Enterprise Technologies, 1, 37-46. 